\title{
Feasibility and implementation of a personalized, web-based exercise intervention for people with cystic fibrosis for 1 year
}

Barlo Hillen ${ }^{1}$, Perikles Simon', Sebastian Schlotter ${ }^{2}$, Oliver Nitsche², Viola Bähner², Krystyna Poplawska ${ }^{2^{*}+}$ and Daniel Pfirmann ${ }^{1+}$

\begin{abstract}
Background: Regular participation in exercise is important for people with cystic fibrosis (CF). Therefore, we implemented a personalized, web-based exercise intervention over the course of one year for people with CF. The aims were to investigate the feasibility of the intervention and to evaluate changes in exercise participation, lung function, and exercise capacity.

Methods: In total, 11/17 participants [aged 12-52 years; FEV \%pred. 72.3 (SD: 17.3)] were included in the final data analysis. Every week, the participants received an individual training recommendation at the start and uploaded their training report on our website at the end of each week. The number of training minutes and sessions performed were analyzed over 13 four-week training sections. The participation in exercise (physical activity questionnaire), lung function and exercise capacity were assessed at baseline (T0), after 12 weeks (T1) and after 52 weeks (T2).

Results: A training duration of $178 \mathrm{~min}$ (SD: 75.5) and 3.3 (SD: 0.89) training sessions could be achieved weekly. In the first four-week training section, the participants performed 137.31 (SD: 95.7) minutes of training, with an increase of $42 \%$ in the third training section (195.01, SD: 134.99). Minutes of training reported on the questionnaire increased by $39.7 \%$ from T0 (179.38 min, SD: 120.9) to T1 (250.63 min, SD: 124.1) but decreased at T2 (166.88, SD: 155.4). There were slight decreases in lung function ( $\mathrm{FEV}_{1}-3.9 \%$ pred.; FVC $-1.9 \%$ pred.) and slight increases in exercise capacity $\left(\mathrm{VO}_{2 \text { peak }}+1.5 \mathrm{ml} / \mathrm{min} / \mathrm{kg}\right.$; six-minute-walk-test-distance $\left.+26 \mathrm{~m}\right)$. Noticeably, five participants experienced deteriorations in their $\mathrm{FEV}_{1}$ of more than $5 \%$ but simultaneously experienced improvements in the parameters of exercise capacity of more than $5 \%$ throughout the year.

Conclusions: The web-based concept was feasible for the participants over the course of a year and supported exercise participation. The improvement in exercise capacity due to increased exercise participation over a prolonged period of time, despite a decrease in lung function, should be further investigated. Finally, if integrated into usual care, this approach could facilitate the prescription of regular personalized exercise and promote exercise participation in the daily lives of people with CF.
\end{abstract}

*Correspondence: Krystyna.Poplawska@unimedizin-mainz.de

${ }^{\dagger}$ Krystyna Poplawska and Daniel Pfirrmann are co-last author

${ }^{2}$ Medical Department of Pediatrics Pulmonology, Allergology and Cystic

Fibrosis, University Medical Centre, Langenbeckstraße 1, 55131 Mainz,

Germany

Full list of author information is available at the end of the article

(c) The Author(s) 2021. Open Access This article is licensed under a Creative Commons Attribution 4.0 International License, which permits use, sharing, adaptation, distribution and reproduction in any medium or format, as long as you give appropriate credit to the original author(s) and the source, provide a link to the Creative Commons licence, and indicate if changes were made. The images or other third party material in this article are included in the article's Creative Commons licence, unless indicated otherwise in a credit line to the material. If material is not included in the article's Creative Commons licence and your intended use is not permitted by statutory regulation or exceeds the permitted use, you will need to obtain permission directly from the copyright holder. To view a copy of this licence, visit http://creativecommons.org/licenses/by/4.0/. The Creative Commons Public Domain Dedication waiver (http://creativeco mmons.org/publicdomain/zero/1.0/) applies to the data made available in this article, unless otherwise stated in a credit line to the data. 
Keywords: Personalized telemedicine, Clinical exercise therapy, Chronic diseases, Exercise prescription, Long-term intervention

\section{Background}

Nowadays, it is well known that exercise performed adequately and regularly has physiological [1], psychological [2] and immunological [3] benefits to human health. Most patients with chronic diseases can benefit from appropriate daily exercise [4]. People with cystic fibrosis (CF) can benefit from regular exercise participation not only in terms of increased respiratory capacity but also the prevention of comorbidities, as the current therapies and new medications have markedly prolonged the lifespans of people with CF [5].

During the past decade, many research groups have observed the positive effects of structured exercise on aerobic physical capacity, health-related quality of life and pulmonary function [6-10]. An improvement in lung function $[11,12]$, and reductions in infection and inflammation [13], the hospitalization rate [14] and fatigue [15] have also been observed due to exercise or physical activity. Ward et al. confirmed that exercise is a fundamental therapeutic intervention for people with CF [10]. Radtke et al. observed no adverse side effects of exercising [8]. Consequently, regular and adequate participation in exercise can be assumed to be an essential part of the lives and management of people with CF.

Nevertheless, evidence regarding the efficacy of exercise training in people with $\mathrm{CF}$ is still limited, and the most effective exercise frequency, intensity, type and time still need to be identified $[8,10]$. Moreover, there is a lack of evidence regarding effective strategies for promoting participation in physical activity at home that is sustained for at least six month by people with CF [16]. Web-based exercise interventions are believed to be a promising solution $[17,18]$.

We developed a personalized, web-based exercise program to promote regular participation in exercise. The use of this web-based concept has already been shown to be feasible for patients with CF over a period of eight weeks [18]. The aim of this study was to examine the feasibility of this web-based exercise intervention and its impact on participation in exercise over the course of an entire year. Additionally, we evaluated self-reported participation in exercise, lung function and exercise capacity before, during and after one year of the intervention.

\section{Methods}

\section{Study design}

This observational, web-based exercise intervention lasted 52 weeks and is a part of the cystic fibrosis online mentoring for microbiome, exercise and diet (COMMED) study. Within COMMED a CF physician, a dietician and a sport scientist could personally interact with the participants via a website and directly at three specific time points. The CF physician was present at each testing timepoint. The dietician could provide nutritional recommendations at each testing timepoint. Whereas the information exchange with the medical doctor and the dietician was voluntary, the participants were obligated to exchange information with the sports scientist weekly. The parameters regarding participation in exercise throughout the intervention were analyzed after 52 weeks. Additionally, we compared self-reported physical activity, lung function and exercise capacity at baseline (T0), after the first 12 weeks (T1) and after 52 weeks (T2).

\section{Participants}

The participants were recruited at the medical Department of Pediatric Pulmonology, Allergology and Cystic Fibrosis of the University Medical Centre in Mainz, Germany. People with CF who met the following criteria were included in the study: age $>12$ years, $\mathrm{FEV}_{1}$ \%pred. $>28$ and $<100$ and/or lung clearance index $(\mathrm{LCI})>9$. The exclusion criteria were orthopedic, rheumatic, cardiovascular or neurological contraindications; no internet access; and an acute exacerbation. Written informed consent was obtained from all participants (or their parents or guardian). The study was approved by the Human Ethics Committee Rhineland-Palatinate (837.288.16 (10, 607)) and conformed to the Code of Ethics of the World Medical Association (Declaration of Helsinki).

\section{Design of the web-based exercise program}

Figure 1 illustrates the design of the weekly feedback loop between the participants and the sports scientist that operated via the website, which is described in the following section.

At baseline, the participants performed an incremental modified walking test (s. Fig. 3) on a treadmill until they reached voluntary maximum exhaustion to identify any exercise-related risks or symptoms, maximum exercise capacity and individual training zones.

Afterwards, the participants were registered on the website and received training material, including three resistance bands (Pinofit ${ }^{\circledR}$ ), a heartrate monitor (Polar FT7), a Redondo ball and two tennis balls. On the website, the participants could download all exercise 


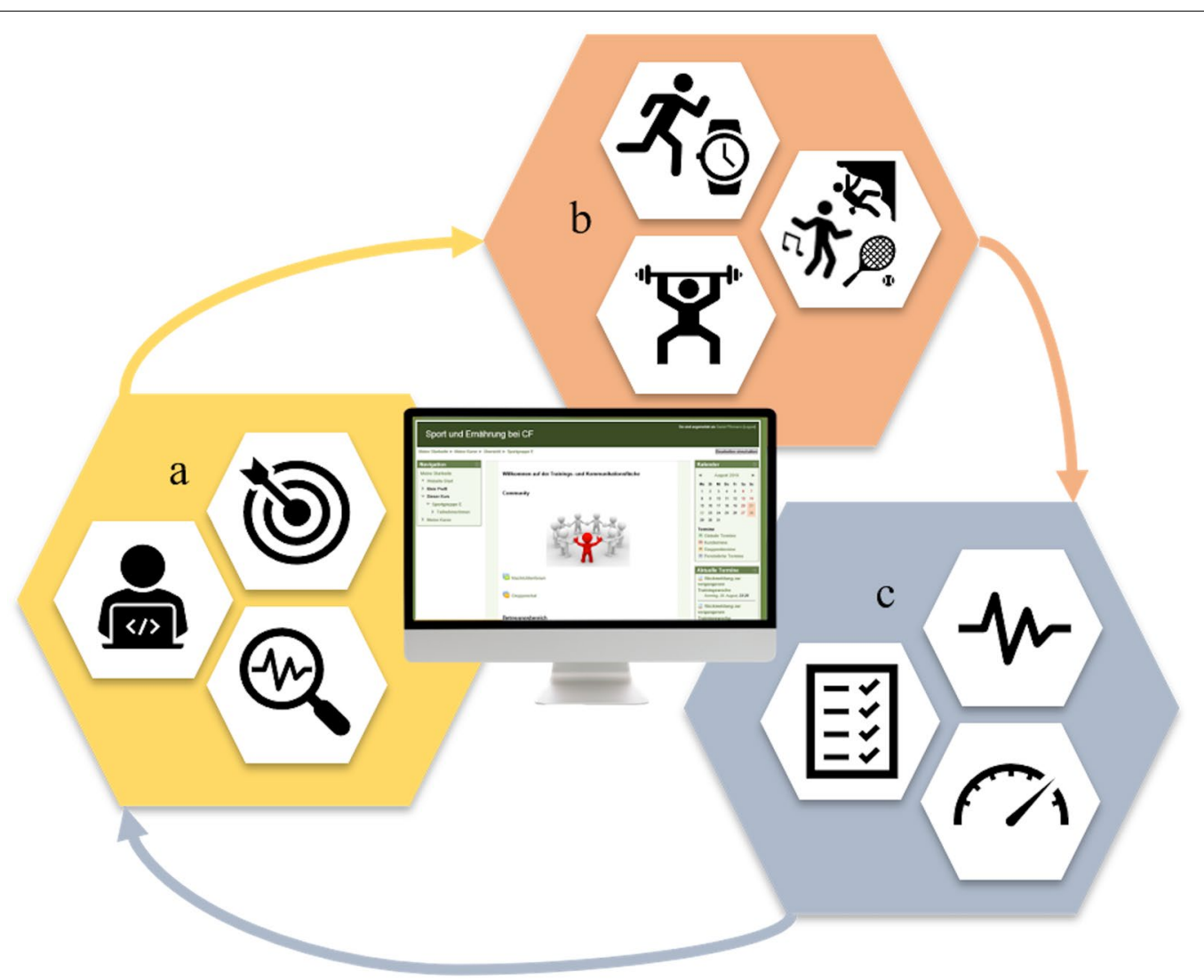

Fig. 1 Design and overview of the weekly feedback loop between the participants and the sports scientist. Legend. The screen in the middle of the figure shows the design of the website. Part a of the concept illustrates the initial and weekly training analysis and individual training adaptation designed by the sports scientist. Part b represents the combined training options, such as endurance training, strength training and preferred physical activities. The blue hexagon illustrates the third essential part of the program, which was feedback provided by the participants to the sports scientist via an uploaded, hand-written exercise protocol at the end of each week. Based on this protocol, the sports scientist could adapt the training load for the following week

descriptions, illustrated manuals or videos; upload their training protocols; receive their weekly training recommendations; and chat with other participants.

At the start of every week, the participants received a message on the website containing a tailored training plan for the following week. This training plan comprised endurance, strength, flexibility and coordination training sessions and integrated preferred physical activities.

Continuous and interval training were recommended for endurance training. The participants could choose their preferred endurance exercise type (brisk walking, running, cycling, rowing, swimming). The recommended exercise intensity was mainly based on the individual anaerobic threshold (IAT) and the variation in oxygen saturation $\left(\mathrm{SpO}_{2}\right)$ measured during the exercise test.

Strength (module 1), coordination (module 2) and flexibility (module 3 ) training sessions were created by the sports scientist and uploaded with descriptions, pictures and videos on the website. Module 1 to 3 could be performed with the training material that had been provided or with everyday objects in the home environment. For instance, the disease-related strength training module consisted of five complete training sessions: (1) "training with own body weight"; (2) "training with free weights or filled water bottles", (3) "training with resistance bands", (4) "training with the Redondo ball", (5) "training on the gymnastic mat". Sets and repetitions of each session and the specific combination of the three modules could be varied and adapted by the sports scientist.

As a third additional option, the participants could pursue their preferred physical activities, such as horseback riding, tennis or climbing.

At the end of each week, the participants had to upload their hand-written exercise protocol (JPEG/PDF) to the website. This exercise protocol contained the performed exercise sessions, the duration of each session and the individual load and discomfort in each session. The individual training load was indicated by the mean heart rate 


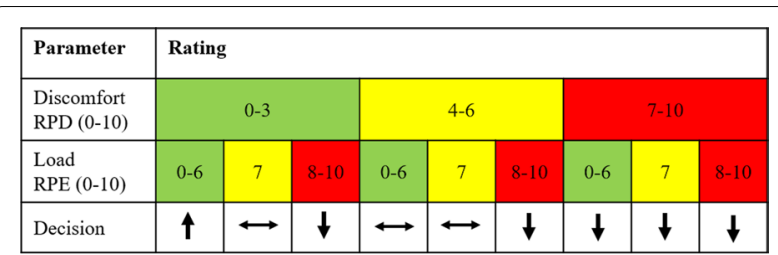

Fig. 2 The decision strategy used to adapt the training load. Legend. After each training session, the participants rated their training session according to the perceived discomfort (0-10) and perceived exertion (0-10). RPD was divided into low (0-3), moderate (4-6) and high (7-10). RPE was also divided into three categories: very light to moderate (0-6), vigorous (7), and hard to maximum effort (8-10). Arrows in the last line illustrate the training load adaptation for each of the nine possible parameter combinations. This decision strategy originated from and the figure is adapted from [18]

per session (HR), measured by the HR monitor, and the rated perceived exertion (RPE 0-10). Rated perceived discomfort (RPD 0-10) was the indicator for exercise tolerance.

After the participants provided feedback via the weekly uploaded training protocol, the sports scientist adapted the exercise program for the upcoming week. Figure 2 shows the decision strategy employed by the sports scientist to adapt the training for the following week. During periods of acute infection or exacerbation or while on holiday, participants could interrupt the training program.

\section{Definition and assessment of exercise participation as primary outcome}

The primary outcome was participation in exercise over the course of one year. The exercise participation parameters were the time spent training and training frequency over 52 weeks for the entire group and for each individual. Therefore, 52 hand-written training protocols for each participant $(n=572)$ were evaluated. Table 1 presents the exercise participation-related parameters.

Additionally, the participants completed the "Physical Activity Questionnaire" (IPAQ) [19], on which they reported their physical activity during the past seven days preceding the intervention (T0), after 12 weeks of the intervention (T1) and after 52 weeks of the intervention (T2). The number of minutes spent training and frequency reported in domain four of the IPAQ, "leisure" (vigorous; moderate), were evaluated.

\section{Assessment of lung function and exercise capacity as secondary outcomes}

Lung function ( $\mathrm{FEV}_{1}, \mathrm{FVC}$ (\%pred.)) and maximal and submaximal exercise capacity $\left(\mathrm{VO}_{2 \text { peak }}\right.$, six-minute walk test distance (6MWTD $(\mathrm{m})$ ), maximum work rate
Table 1 Evaluated parameters of exercise participation

\begin{tabular}{ll}
\hline Nr. & Exercise participation parameter \\
\hline 1.1 & Individual training time/week (mean_min) \\
1.2 & Individual training sessions/week (mean_sessions) \\
1.3 & Individual training weeks (n/52; \%) \\
2.1 & Group training time/training section ${ }^{\text {a }}$ (mean_min) \\
2.2 & Group training sessions/training section (mean_sessions) \\
3.1 & Domain 4 IPAQ_leisure (moderate, vigorous): training minutes at \\
& T0, T1,T2 \\
3.2 & Domain 4 IPAQ_leisure (moderate, vigorous): training sessions at \\
& T0, T1, T2
\end{tabular}

a Section: The 52 weeks of the intervention were divided into 13 4-week training sections

$\left(\operatorname{Power}_{\text {peak }}(\mathrm{w})\right)$, work rate at the individual anaerobic threshold (Power IAT $\left._{(\mathrm{w})}\right)$, and heart rate at the individual anaerobic threshold $\left(\mathrm{HR}_{\mathrm{IAT}}\right.$ (beats/min)) were the secondary outcomes. These parameters were measured at baseline (T0), after 12 weeks (T1) and after 52 weeks (T2) of the intervention.

Forced expiratory volume in one sec $\left(\mathrm{FEV}_{1}\right)$ and forced vital capacity (FVC) (\%pred.) were assessed using flowvolume spirometry (MasterScope ${ }^{\circledR}$ Body, CareFusion, Germany) in accordance with the criteria of the American Thorax Society/European Respiratory Society. FEV and FVC were determined as the highest of three acceptable measurements and expressed as a percentage of the predicted reference value according to the Global Lung Function Initiative [20].

The 6-min walk test (6MWT) was conducted to assess submaximal exercise capacity.

The 6MWTD was the output of the 6MWT. The participants had to achieve their maximum distance within 6 min while moving at their fastest possible walking speed.

The cardiopulmonary exercise test (CPET), including a lactate analysis, was performed to assess submaximal and maximal exercise capacity. A modified incremental walking test protocol [21] was performed on a treadmill (Saturn, HP Cosmos ${ }^{\circledR}$ ) with increasing speed $(\mathrm{km} / \mathrm{h})$ and incline (\%) until maximum exhaustion was reached (s. Fig. 3). The increments lasted three minutes.

Ventilation of $\mathrm{O}_{2}\left(\mathrm{VO}_{2}\right)$ and $\mathrm{CO}_{2}\left(\mathrm{VCO}_{2}\right)$ (Ergostick, Geratherm $^{\circledR}$ ), lactate (L) (EKF diagnostics ${ }^{\circledR}$; Winlactat 3.0 Mesics $\mathrm{GmbH}$ ), heart rate (HR) (ECGpro, Amed$\left.\mathrm{Tec}^{\circledR}\right)$, peripheral capillary oxygen saturation $(\mathrm{SpO} 2)$ and rate of perceived exertion (RPE) [22] were measured during the $6 \mathrm{MWT}$ and the CPET. L samples were taken from the ear lobe after each increment of the CPET. The individual anaerobic threshold (IAT) was determined according to the Dickhuth model [23]. $\mathrm{VO}_{2 \text { peak }}$ was determined to be highest $\mathrm{VO}_{2}$ during the last $30 \mathrm{~s}$ of the CPET. 


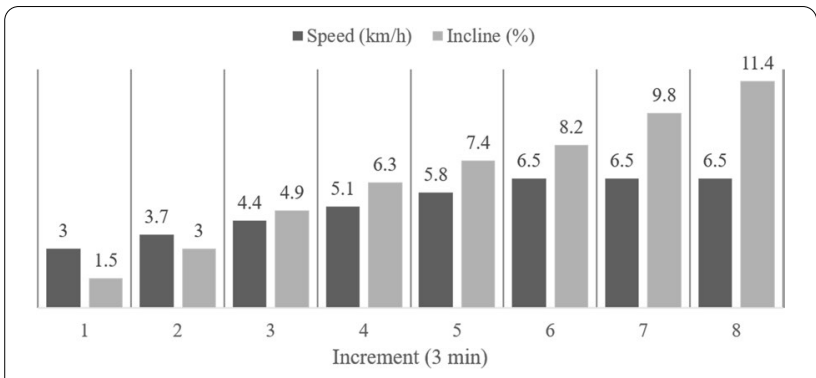

Fig. 3 Modified walking test protocol performed on a treadmill during cardiopulmonary exercise testing

\section{Statistical analysis}

Microsoft Excel (2013) and IBM SPSS Statistics version 23.0 were utilized. We analyzed the exercise participation data exploratively (mean, standard deviation, median, min, max, range, 95\% confidence intervals). We performed repeated-measures ANOVA for the analysis of the primary and secondary outcomes. To test the violation of the assumption of a normal distribution, the Shapiro Wilk-test was conducted. Mauchly's W-test was performed to test for sphericity. The Greenhouse-Geisser correction $(\varepsilon)$ was applied when the assumption of sphericity was not met. The significance level of $p<0.05$ was set for all statistical analyses.

\section{Results}

Initially, 17 participants were included in this study. Six of those participants dropped out, and 11 participants were included in the final data analysis for the entire year. This participant group had a mean age of 32.9 years $(\mathrm{SD} \pm 11.4)$, mean FEV1\%pred. of 72.3 $(\mathrm{SD} \pm 17.3)$ and a mean BMI of $22.7 \mathrm{~kg} / \mathrm{m}^{2}(\mathrm{SD} \pm 2.18)$ (Table 2). Of the 11 included subjects, six were males and five were females. The dropout rate of $35.3 \%$ occurred for the following reasons: no response after three weeks $(n=1)$, difficulties with changing life circumstances $(n=1)$, severe pulmonary exacerbation $(n=1)$, infections immediately before T2 $(n=2)$ and acute kidney failure $(\mathrm{n}=1)$.

\section{Exercise participation throughout the year}

The weekly exchange between the sports scientist and each participant remained feasible throughout the year. Communication was interrupted briefly during vacations and in some cases due to inpatient stays. The number of training sessions and the training duration in minutes were extracted from 572 handwritten training protocols. Table 2 and Fig. 4 show the descriptive analysis of the groups' exercise participation during the 52 weeks of the intervention.
Table 2 Overview of the parameters of exercise participation

\begin{tabular}{|c|c|c|c|}
\hline$(\mathrm{N}=11)$ & $\begin{array}{l}\text { Training weeks } / 52 ; \\
\%^{\mathrm{a}} \text { (SD) }\end{array}$ & Training time/week ${ }^{b}$ & $\begin{array}{l}\text { Training } \\
\text { sessions/ } \text { week }^{c}\end{array}$ \\
\hline Mean & 40.2 (7.49); $77.3 \%(14.8)$ & $178(75.5)$ & $3.32(0.886)$ \\
\hline Median & $41 ; 79 \%$ & 182 & 3.32 \\
\hline Range & $28 ; 53 \%$ & 249 & 3.30 \\
\hline Min & $23 ; 45 \%$ & 59.9 & 1.48 \\
\hline Max & $51 ; 98 \%$ & 309 & 4.78 \\
\hline
\end{tabular}

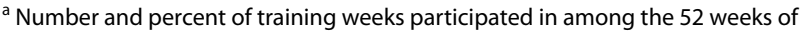
the intervention

${ }^{b}$ Training time $(\mathrm{min})$ per active training week within the intervention

${ }^{\mathrm{c}}$ Training sessions per week within the active training weeks

Subjects 8 and 11 achieved the most exercise training time ( $>300 \mathrm{~min} /$ week), most training frequency ( $>4$ sessions/week) and participated in $85 \%$ [8] and $88 \%$ [11] of the predetermined training weeks. To achieve this high level of exercise participation, subject 8 chose hiking and climbing as the main activities, whereas subject 11 preferred swimming, running, cycling, resistance exercise and horseback riding. Therefore, subject 8 had a higher overall exercise intensity than subject 11 had.

For the analysis of the variation in exercise participation throughout the year, the 52 weeks were divided into 13 four-week training sections. In the first training section, the participants participated in 137.31 (SD: 95.7) training minutes. In all of the following training sections, the number of minutes spent training were. The highest mean numbers of training minutes were achieved in the third (195.01, SD: 134.99), seventh (210.7, SD: 104.56) and tenth (205.18, SD: 125.72) training sections. From the first four-week (1-4) section to the third four-week section (9-12), the number of training minutes increased by $42 \%$. Figure 5 shows the mean training minutes achieved for each training section.

In the first training section, the participants participated in 3.2 (SD: 1.4) training sessions. Only in section nine was the number of mean weekly training sessions lower. The maximum numbers of training sessions occurred in training sections three (3.5, SD: 0.9), six (3.5, SD: 1.3) and seven (3.4, SD: 1.3). Figure 6 shows the training sessions completed in each training section.

The mean numbers of minutes spent training and training sessions in each for the 13 four-week training sections for the seven participants could be included in the repeated-measures ANOVA due to the listwise exclusion of missing data. Missing data resulted from training sections for which no mean value could be calculated for a participant, because in at least one of the four weeks the training was interrupted. The data were normally distributed at all timepoints $(p>0.05)$ except in training 


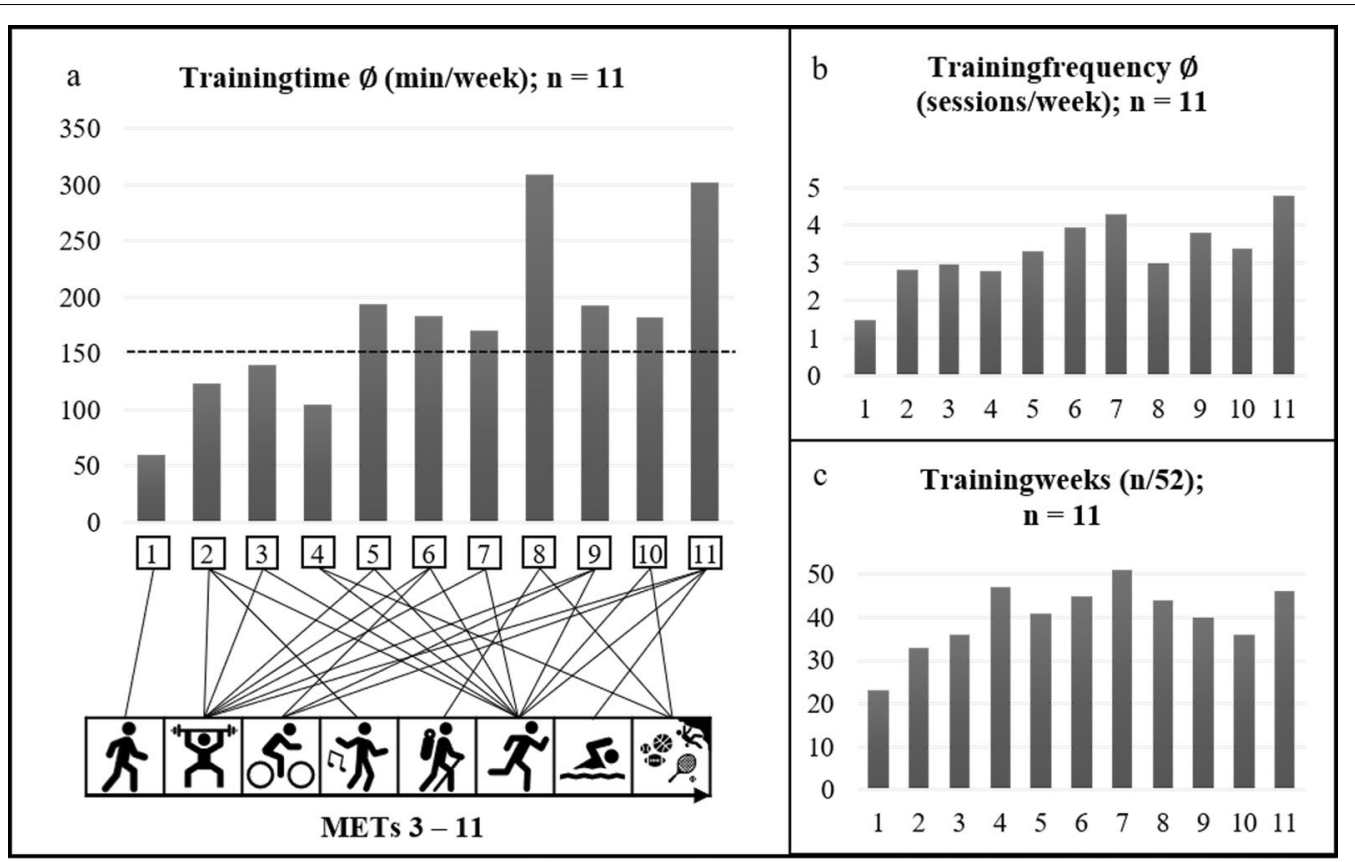

Fig. 4 Exercise participation of each participant over the course of the 52 weeks of the intervention. Legend. a illustrates training time (min/week) in combination with performed exercise types and METs for each participant. METs, metabolic equivalents $(1 \mathrm{MET}=3.5 \mathrm{male} ;=3.15 \mathrm{VO} 2(\mathrm{ml} / \mathrm{min} /$ $\mathrm{kg})$ ). METs for each exercise type are in accordance with [24]. b Illustrates the mean performed training sessions per week during the intervention. $\mathbf{c}$ Shows the active training weeks from the intended 52 week of intervention

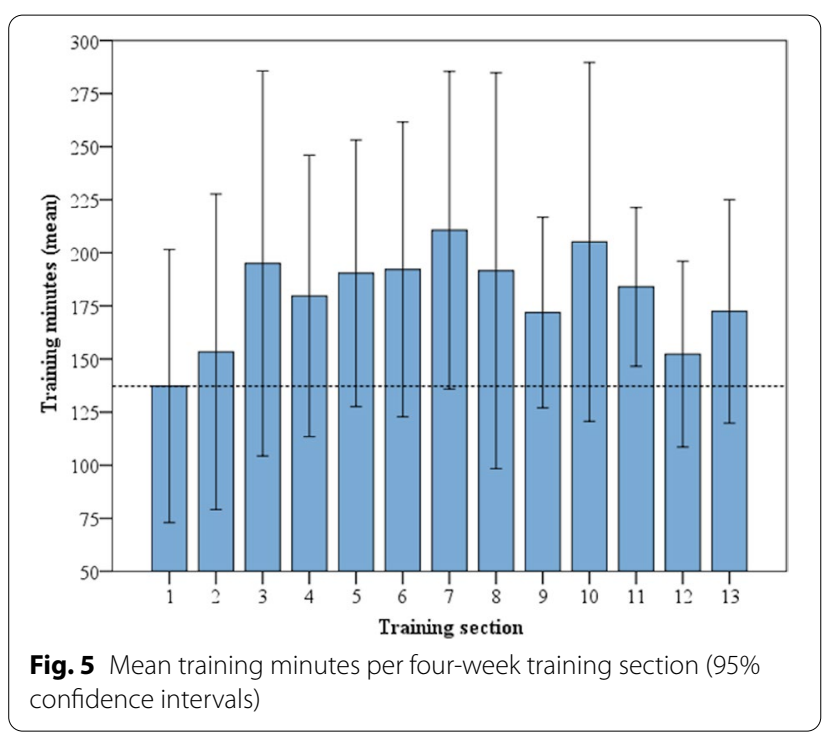

section $2(p=0.01)$ for the number of minutes spent training. Sphericity was not observed $(p=0.000)$ between the training sections. Greenhous Geisser correction was applied. Repeated-measures ANOVA revealed no significant difference between the training sections with

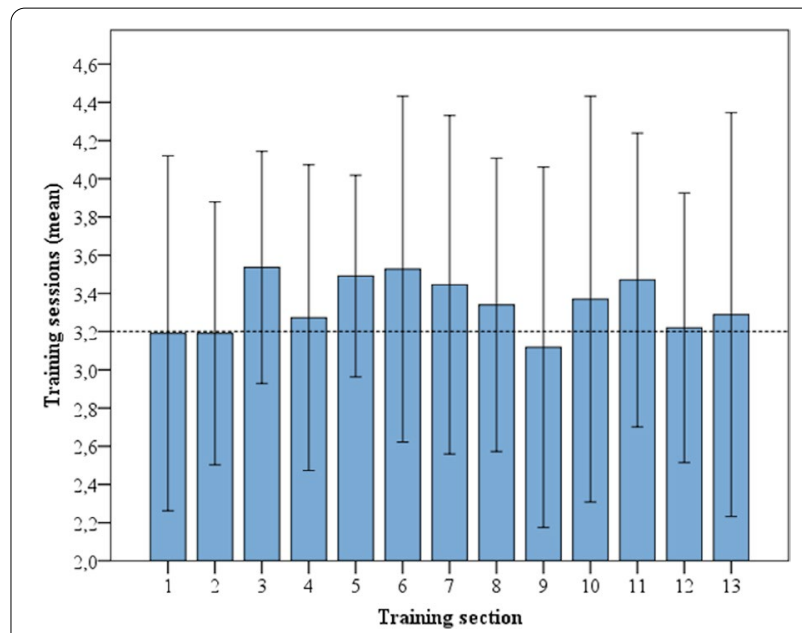

Fig. 6 Mean training sessions per four-week training section (95\% confidence intervals)

regard to training minutes $(p=0.366)$ or training sessions $(p=0.478)$. The distinct range of the confidence intervals, i.e., the heterogeneous participation in training in the group, led to the non-significant differences between each training section. 


\section{Self-reported physical activity at T0, T1, T2}

The self-reported physical activity of eight participants who completed the IPAQ at T0, T1 and T2, could be included in the repeated measures ANOVA due to the listwise exclusion of missing data. Missing data resulted from the absence of information provided in the questionnaire at least at one timepoint $(3 / 33)$. The data were normally distributed at each timepoint $(p>0.05)$, and sphericity was observed $(p=0.806)$. These 8 participants reported a mean of $179.38 \mathrm{~min}$ (SD: 120.9 ) at T0, $250.63 \mathrm{~min}$ (SD: 124.1) at T1, and 166.88 (SD: 155.4) at T2. There was no significant difference between the timepoints $(p=0.120)$. The self-reported number of minutes spent training increased by $39,7 \%$ from $\mathrm{T} 0$ to
T1. This identified increase was no longer evident after 52 weeks.

\section{Lung function and exercise capacity at T0, T1, T2}

From T0 to T2, there were slight decreases in the mean $\mathrm{FEV}_{1}-3.9 \%$ pred. $(p=0.102)$ and in the $\mathrm{FVC}$ of $-1,9 \%$ pred. $(p=0.575)$; slight increases in the $\mathrm{VO}_{2 \text { peak }}$ of $+1.5 \mathrm{ml} / \mathrm{min} / \mathrm{kg}(p=0.413)$, the 6 MWTD of $+26 \mathrm{~m}$ $(p=0.287)$ and the Power $_{\text {peak }}$ of $+0.11 \mathrm{w} / \mathrm{kg}(p=0.362)$; and slight decrease in the Power $_{\mathrm{IAT}}$ of $-0.01(p=0.714)$ and the $\mathrm{HR}_{\mathrm{IAT}}$ of $-4 \mathrm{~b} / \mathrm{min}(p=0.230)$ (Table 3$)$.

There were heterogeneous changes in the secondary outcomes in the interindividual comparison. The changes in exercise capacity are shown in Fig. 7. Figure 8 shows

Table 3 Comparison of exercise capacity and lung function between timepoints

\begin{tabular}{|c|c|c|c|c|c|c|c|c|}
\hline & TP & FEV $^{a}$ & FVC & $\mathrm{VO}_{\text {peak }}{ }^{\mathrm{b}}$ & 6MWTD & Power $_{\text {peak }}$ & Power $_{\text {IAT }}$ & $\mathrm{HR}_{\mathrm{IAT}}$ \\
\hline \multirow[t]{3}{*}{ Mean (SD) } & TO & $72.3(17.3)$ & $87.3(13.4)$ & $28.5(6.5)$ & $590(87.1)^{a}$ & $1.51(0.5)$ & $1.13(0.3)$ & $148(13.3)$ \\
\hline & $\mathrm{T} 1$ & 71.0 (19.8) & $85.6(13.7)$ & $29.4(7.0)$ & $601(62.3)$ & $1.56(0.5)$ & $1.14(0.4)$ & 147 (11.6) \\
\hline & $\mathrm{T} 2$ & $68.4(20.8)$ & $85.4(15.6)$ & $30.0(9.0)$ & $616(56.3)$ & $1.62(0.6)$ & $1.12(0.3)$ & $144(10.9)$ \\
\hline rmANOVA (p) & & 0.102 & 0.575 & 0.413 & $0.287^{b}$ & $0.362^{b}$ & $0.714^{b}$ & 0.230 \\
\hline
\end{tabular}

TP, time point; T0, baseline assessment; T1, assessment after 12 weeks; T2, assessment after 52 weeks; FEV 1 , forced expiratory volume in one second (\%predicted); FVC, forced vital capacity (\%predicted); $\mathrm{VO}_{2}$ peak, oxygen uptake at maximum work rate ( $\mathrm{ml} / \mathrm{min} / \mathrm{kg}$ ); $6 \mathrm{MWTD}$, six-minute walk test-distance (m); Power ${ }_{\text {peak }}$, maximum work rate $(\mathrm{w} / \mathrm{kg})$; Power $_{\mathrm{IAT}}$, work rate at individual anaerobic threshold; $\mathrm{HR}_{\mathrm{IAT}}=$ heartrate (beats/min); SD, standard deviation; rmANOVA, repeated-measures analysis of variance

${ }^{\text {a }}$ Data violated the assumption of a normal distribution

${ }^{\mathrm{b}}$ Greenhouse-Geisser correction was applied

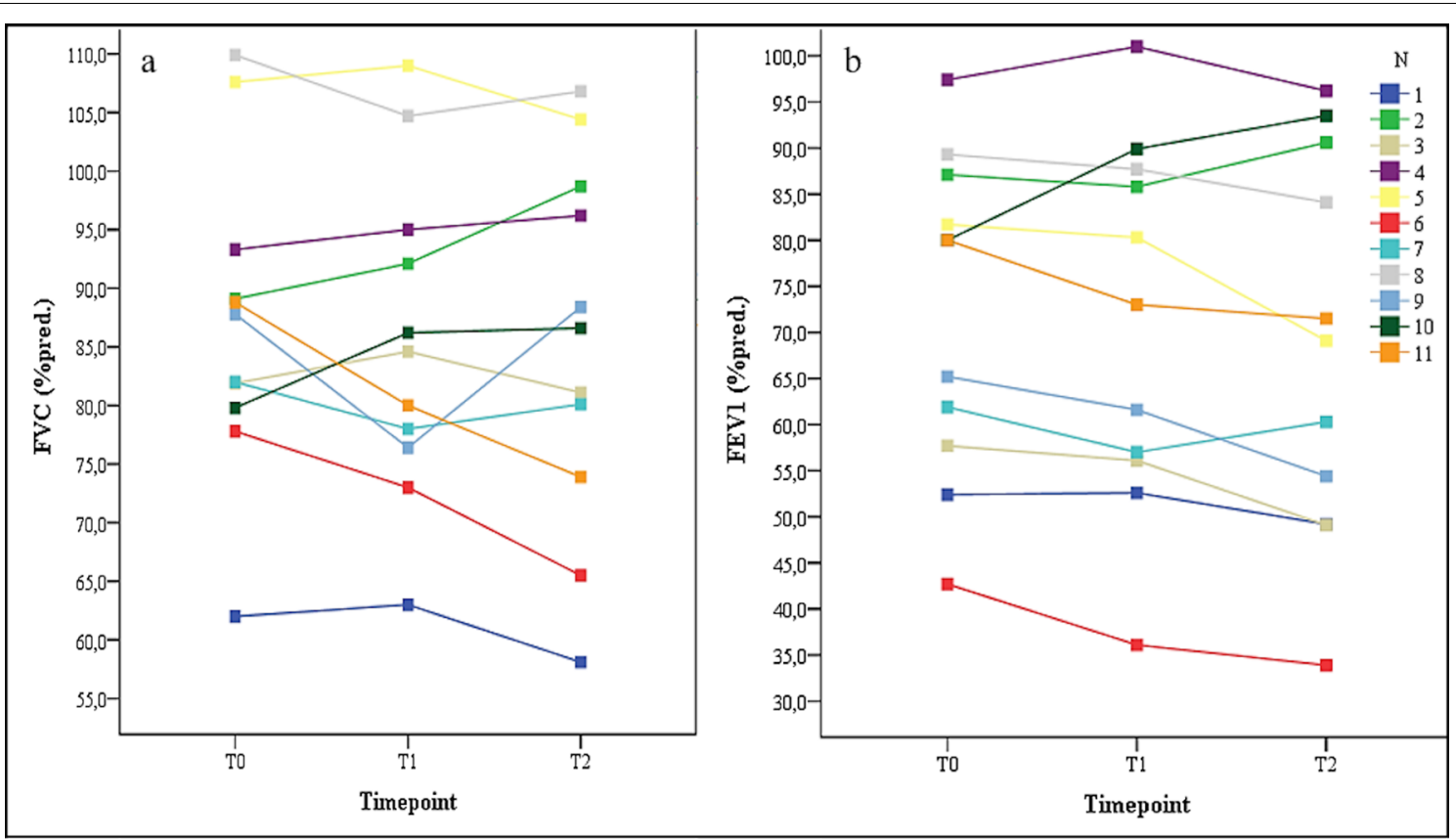

Fig. 7 Changes in $\mathrm{VO}_{2 \text { peak }}$ and 6MWTD from T0 to T1 to T2 for each participant. Legend. T0, baseline assessment; T1, assessment after 12 weeks; T2, assessment after 52 weeks; $V_{2}$ peak $=$ oxygen ventilation at maximum work rate $(\mathrm{ml} / \mathrm{min} / \mathrm{kg}) ; 6 \mathrm{MWTD}=$ six-minute walk test distance (m) 

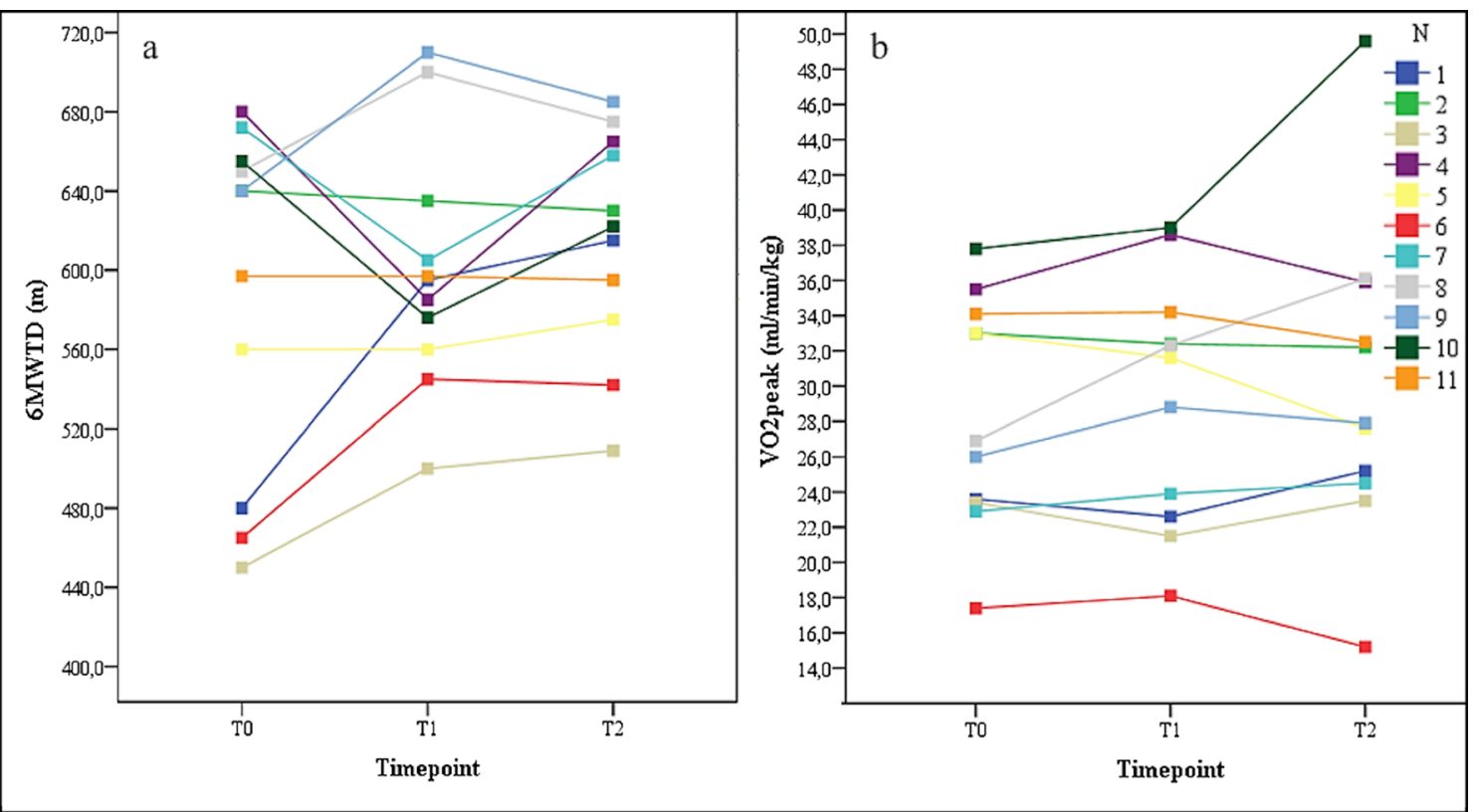

Fig. 8 Changes in $\mathrm{FEV}_{1}$, and FVC from T0 to T1 to T2 for each participant. Legend. T0, baseline assessment; T1, assessment after 12 weeks; T2, assessment after 52 weeks; $\mathrm{FEV}_{1}=$ forced expiratory volume in one second (percent predicted); $\mathrm{FVC}=$ forced vital capacity (percent predicted)

the changes in lung function for each participant across all tests. Seven participants increased their 6MWTD, seven increased their $\mathrm{VO}_{2 \text { peak }}$, nine decreased their $\mathrm{FEV}_{1}$ and seven decreased their FVC.

Under the exploratory assumption that a five percent change in pulmonary function and exercise capacity from T0 to T2 could be considered clinically relevant, the following observations can be made: Subjects 1, 3, 6, and 9 improved their $6 \mathrm{MWTDs}$ by more than $5 \%$. Only participant 10 experienced a worsening of the 6MWTD by slightly more than $5 \%$. Participants $1,7,8,9$, and 10 improved their $\mathrm{VO}_{2}$ peak by more than $5 \%$. Participants 5 and 6 experienced decreases in their $\mathrm{VO}_{2}$ peak of more than $5 \%$. Only participant 10 improved the $\mathrm{FEV}_{1}$ by more than $5 \%$. Participants $1,3,5,6,8,9$, and 11 experienced reductions in their $\mathrm{FEV}_{1}$ of more than 5\%. Participants 1 , $2,3,4,5$, and 10 improved their FVC by more than $5 \%$. No participant experienced a reduction in the FVC. Considering the individual changes in secondary outcomes as a whole, it is interesting that participants $1,3,6,8$, and 9 experienced reductions in their $\mathrm{FEV}_{1}$ by more than $5 \%$ but experienced improvements in the parameters of exercise capacity of more than $5 \%$.

\section{Discussion}

Taking into account that according to the $\mathrm{WHO}$ guidelines for physical activity, $150 \mathrm{~min} /$ week is recommended for healthy adults [25] and that overall adherence of young people to exercise recommendations is generally poor, the overall achieved mean training time of $178 \mathrm{~min}$ per week is noteworthy. The mean training time was lower in the first four-week section $(<150 \mathrm{~min} /$ week $)$ than in the following 12 training sections $(>150 \mathrm{~min} /$ week). This means that a sustained increase in training minutes can be assumed to be indicative of the long-term suitability impact of the intervention.

However, a progressive increasing trend in exercise participation could be observed only until the seventh four-week training section. In the following training sections, exercise participation fluctuated. These fluctuations could be related to the normal health changes in people with CF that occur over the course of a year or to reduced reporting. The exercise participation protocol had to be written by hand and then uploaded to the website as a scan or image. This appeared to be more a result of the circumstances of the study than a necessary element of the program, and the automatic acquisition of training data, and the transformation of the web-based concept into a mobile application could solve this issue in the future. Moreover, fluctuations in exercise participation could also be due to the time of year, as infections are more common in colder months and engagement in physical outdoor activities usually decreases somewhat during those months as well. Additional indoor training opportunities at home such as endurance training on an ergometer might support exercise participation during the colder periods of the year. 
As shown in the results, the self-reported number of minutes spent training and sessions in domain 4 of the IPAQ were also higher at T1 than before the intervention, which strengthens the assumption that the exercise intervention could support exercise participation in people with CF. Notably, the exercise participation assessed at T0 and T1 on the IPAQ was higher than the reported exercise participation reported in our protocols. However, the percentage increase in exercise participation from T0 to T1 (IPAQ) or from the first training section to the third training section (exercise protocol) was similar. The potential overreporting on the IPAQ has also been reported previously [26]. An automated, objective measurement of exercise participation utilizing reliable wearables might further verify this observation in future studies. At T2, the exercise participation reported on the IPAQ was lower than that at $\mathrm{T} 1$, which might attenuate the long-term suitability of the intervention.

Considering these observations, the long-term feasibility of the web-based concept is clear, and exercise participation did increase, especially during the first three to seven month of the intervention; however, the long-term suitability ( $>7$ month) should be further investigated.

Remarkably, despite slight increases in $\mathrm{VO}_{2 \text { peak }}$ and 6MWTD, slight decreases in $\mathrm{FEV}_{1}$ and FVC occurred after our intervention. Even a "stabilization" of these parameters over the course of a year in people with progressive disease [27] would be considered a positive outcome. Most interestingly, five participants experienced reductions in their lung function but enhancements or no changes in their exercise capacity. However, we cannot assume that this "stabilization" occurred only due to our intervention. One reason is the lack of a control group and potentially influential factors, such as medication, which come into play during a one-year intervention under real-world conditions. A recent study, which evaluated a large international sample of people with CF, confirmed the importance of exercise capacity as a predictor for long-term mortality [28]. In this context, even a small increase in exercise capacity, despite an observed reduction in lung function in our study, may have a positive effect on long-term survival in our participants. However, while we have now shown the practicability of a long-term web-based approach, the concrete health effects need to be determined.

There are other reports of telehealth approaches to the management of CF [28-33]. Cox et al. [29] demonstrated the feasibility and acceptability of the internet-based program "Active Online", which lasted eight weeks, in combination with phone calls every second week in ten adults with CF (mean age 30 years and mean FEV $50 \%$ pred.). They suggested conducting a randomized controlled trial to examine the effect on participation in physical activity.
In 2018, Chen et al. [30] addressed the issue of the risk of cross-infection in the population of CF patients. Therefore, they examined the use of a live-streaming platform that offered supervised and interactive group exercises. In this examination, ten patients had to perform $30 \mathrm{~min}$ of aerobic resistance training three times per week for six weeks. The patients completed $85 \%$ of the training sessions, and they reported enjoying the training and gave the platform a high rating. The effects on physiological parameters were not reported. The effectiveness of a home-based video game program for young CF patients was tested by Del Corral et al. [31]. The research group observed that six weeks of the video game intervention during rehabilitation improved the participants' sixminute walk test distance and health-related quality of life, among other parameters. The patients completed a 30- to 60-min training session five days per week, but they included very young patients (mean 13 years old) with a mean $\mathrm{FEV}_{1}$ of $82.7 \%$ predicted. A year later, Tomlinson et al. [34] presented the concept of "video calling via skype". Nine patients completed eight weeks of this intervention, during which an exercise therapist supervised all sessions. They achieved $68 \%$ compliance but also reported no changes in the health parameters. All of these studies were short-term interventions.

Recently, elaborate and promising study protocols for web-based RCTs have been published. Hebestreit et al. [30] published a study protocol for "Activate-CF" involving a "partially supervised conditioning program" based on the use of pedometers, with an international, multicenter randomized controlled trial design. Lang et al. [31] presented a study protocol for a 12-months multimodal telehealth-based outpatient physiotherapy service and compare the usual outpatient physiotherapy service with the telehealth intervention CyFiT, eHAB ${ }^{\circledR}$. This software allows real-time video sharing, the measurement of motion in various joint, and virtual group-based sessions combined with the use of an activity tracker. Therefore, it remains to be determined how these approaches affect exercise participation in people with CF and influence their lung function and exercise capacity. Innovative person-centered, web-based algorithms aiming in automatically increase and measure physical activity, set personalized physical activity goals, and determine the type and duration of physical activity [35] could also be considered in the design of future studies.

\section{Limitations}

Some limitations of our study must be taken into account. The most important is the small sample size and its heterogeneity due to the inclusion of participants at every stage of CF. This limitation, combined with a lack of a control group, potentially attenuates the power of 
the reported p-values, which should rather be considered as a supplement to the performed exploratory analysis. A general estimation of the most effective exercise type, duration, intensity and frequency for people with $\mathrm{CF}$ cannot be derived from the results. Additionally, over the course of an entire year, our participants needed continuous changes in medications and suffered from acute infections or pulmonary exacerbations, which influenced the outcome. The analysis of exercise participation was based on self-reported protocols. Factors such as social desirability or obliviousness could have caused reporting bias. Nevertheless, self-reporting allows a mentor to receive individual feedback from participants about their current perceptions, efforts and training progression. The recruitment of more participants, the inclusion of a control group, the automation of training data acquisition, the measurement of preintervention exercise participation and the restriction of the exercise options could help overcome the above-mentioned limitations in future studies.

\section{Conclusions}

The web-based concept was feasible for the participants over the course of an entire year and supported their exercise participation. The weekly feedback loop between participants and the sports scientist enabled the provision of regular personalized exercise counselling. Automatic acquisition of the training data and a mobile application could further improve this web-based concept. The potential increase in exercise capacity due to increased participation in exercise over a prolonged period of time, despite a decrease in lung function, should be further investigated. Finally, if integrated into usual care, this approach could facilitate the prescription of regular personalized exercise and promote exercise participation in the daily lives of people with CF.

\begin{abstract}
Abbreviations
CF: Cystic fibrosis; COMMED: Cystic fibrosis online mentoring for microbiome exercise and diet; CPET: Cardiopulmonary exercise testing; FEV 1 : Forced expiratory volume in one sec; FVC: Forced vital capacity; HR: Heartrate; $H R_{I A T}$; Heartrate at individual anaerobic threshold; IAT: Individual anaerobic threshold; IPAQ: International physical activity questionnaire; L: Lactate; LCI: Lung clearance index; Power ${ }_{\mid A T}$ : Watt at individual anaerobic threshold; Power peak: Watt at maximum exhaustion during cardiopulmonary exercise testing; $\mathrm{S}_{\mathrm{p}} \mathrm{O}_{2}$ Peripheral capillary oxygen saturation; RPE: Rate of perceived exertion; RPD: Rate of perceived discomfort; T0: Baseline; T1: After 12 weeks of the intervention; T2: After 52 weeks of the intervention; $\mathrm{VCO}_{2}$ : Ventilation of $\mathrm{CO}_{2} ; \mathrm{VO}_{2}$ : Ventilation of $\mathrm{O}_{2} ; \mathrm{VO}_{2 \text { peak }}$ : Highest $\mathrm{VO}_{2}$ during the last $30 \mathrm{~s}$ of the CPET.
\end{abstract}

\section{Acknowledgements}

We are very thankful to the people with CF that voluntary participated in this study.

\section{Authors' contributions}

$\mathrm{DP}, \mathrm{KP}, \mathrm{PS}, \mathrm{BH}$ designed the study. $\mathrm{BH}, \mathrm{DP}, \mathrm{KP}$ interpreted the data. $\mathrm{BH}, \mathrm{DP}, \mathrm{KP}$, prepared the manuscript. All authors reviewed the manuscript and approved the final version of the manuscript before submission.

\section{Funding}

Open Access funding enabled and organized by Projekt DEAL. The authors gratefully acknowledge funding support for this work from Grant Vertex: CFCircle of Care.

\section{Availability of data and materials}

The datasets generated and/or analyzed during the current study are not publicly available because they contain sensitive patient data but are available from the corresponding author upon reasonable request.

\section{Declarations}

Ethics approval and consent to participate

Written informed consent was obtained from all participants (or their parents or guardian). The study was approved by the Human Ethics Committee Rhineland-Palatinate (837.288.16 (10607)) and conformed to the Code of Ethics of the World Medical Association (Declaration of Helsinki).

\section{Consent for publication}

Not applicable.

\section{Competing interests}

The authors declare that they have no competing interests.

\section{Author details}

${ }^{1}$ Department of Sports Medicine, Disease Prevention and Rehabilitation, Johannes Gutenberg University, Mainz, Germany. ${ }^{2}$ Medical Department of Pediatrics Pulmonology, Allergology and Cystic Fibrosis, University Medical Centre, Langenbeckstraße 1, 55131 Mainz, Germany.

Received: 16 December 2020 Accepted: 29 July 2021

Published online: 19 August 2021

\section{References}

1. Pedersen BK, Saltin B. Exercise as medicine-evidence for prescribing exercise as therapy in 26 different chronic diseases. Scand J Med Sci Sport. 2015;25:1-72.

2. Mikkelsen K, Stojanovska L, Polenakovic M, Bosevski M, Apostolopoulos V. Exercise and mental health. Maturitas. 2017;106:48-56.

3. Alack K, Pilat C, Krüger K. Current knowledge and new challenges in exercise immunology. Dtsch Z Sportmed. 2019;70(10):250-60.

4. Pedersen BK. Physical exercise in chronic diseases. Nutr Skeletal Muscle. 2019;217-66.

5. Regard L, Lafoeste H, Martin C, Chassagnon G, Burgel PR. Ageing with cystic fibrosis: classical and emerging comorbidities in adults with cystic fibrosis. Rev Pneumol Clin. 2018;74(5):279-91.

6. Cox NS, Elkins MR. Physical training has several benefits for people with cystic fibrosis. Br J Sports Med. 2011;45(10):835-6.

7. Hebestreit $\mathrm{H}$, Kriemler S, Radtke T. Exercise for all cystic fibrosis patients: Is the evidence strengthening? Curr Opin Pulm Med. 2015;21(6):591-5.

8. Radtke T, Nevitt S, Hebestreit H, Kriemler S. Physical exercise training for cystic fibrosis. Cochrane Database Syst Rev. 2017;11(11):1-145.

9. Vendrusculo FM, Heinzmann-Filho JP, da Silva JS, Perez Ruiz M, Donadio MVF. Peak oxygen uptake and mortality in cystic fibrosis: systematic review and meta-analysis. Respir Care. 2019;64(1):91-8.

10. Ward N, Stiller K, Holland AE. Exercise as a therapeutic intervention for people with cystic fibrosis. Expert Rev Respir Med. 2019;13(5):449-58.

11. Paranjape SM, Barnes LA, Carson KA, von Berg K, Loosen H, Mogayzel PJ. Exercise improves lung function and habitual activity in children with cystic fibrosis. J Cyst Fibros. 2012;11(1):18-23. 
12. Kriemler S, Kieser S, Junge S, Ballmann M, Hebestreit A, Schindler C, et al. Effect of supervised training on FEV1 in cystic fibrosis: a randomised controlled trial. J Cyst Fibros. 2013;12(6):714-20.

13. Van de Weert-van Leeuwen PB, Arets HGM, van der Ent CK, Beekman JM. Infection, inflammation and exercise in cystic fibrosis. Respir Res. 2013;14(32):1-10.

14. Vandekerckhove K, Keyzer M, Cornette J, Coomans I, Pyl F, De Baets F, et al. Exercise performance and quality of life in children with cystic fibrosis and mildly impaired lung function: relation with antibiotic treatments and hospitalization. Eur J Pediatr. 2017;176(12):1689-96.

15. Orava C, Fitzgerald J, Figliomeni S, Lam D, Naccarato A, Szego E, et al. Relationship between physical activity and fatigue in adults with cystic fibrosis. Physiother Can. 2018;70(1):42-8.

16. Cox NS, Alison JA, Holland AE. Interventions to promote physical activity in people with cystic fibrosis. Paediatr Respir Rev. 2014;15(3):237-9.

17. Cox NS, Alison JA, Rasekaba T, Holland AE. Telehealth in cystic fibrosis: a systematic review. J Telemed Telecare. 2012;8(2):72-8.

18. Pfirrmann D, Haller N, Huber Y, Jung P, Lieb K, Gockel I, et al. Applicability of a web-based, individualized exercise intervention in patients with liver disease, cystic fibrosis, esophageal cancer, and psychiatric disorders: process evaluation of 4 ongoing clinical trials. JMIR Res Protoc. 2018;20(5):1-19.

19. Craig CL, Marshall AL, Sjöström M, Bauman AE, Booth ML, Ainsworth BE, et al. International physical activity questionnaire: 12-country reliability and validity. Med Sci Sports Exerc. 2003;35(8):1381-95.

20. Quanjer PH, Stanojevic S, Cole TJ, Baur X, Hall GL, Culver BH, et al. Multiethnic reference values for spirometry for the 3-95-yr age range: the global lung function 2012 equations. Eur Respir J. 2012;40(6):1324-43.

21. Pfirrmann D, Tug S, Brosteanu O, Mehdorn M, Busse M, Grimminger PP, et al. Internet-based perioperative exercise program in patients with Barrett's carcinoma scheduled for esophagectomy [iPEP-study] a prospective randomized-controlled trial. BMC Cancer. 2017;17(413):1-8.

22. Borg G. Perceived exertion as an indicator of somatic stress. Scand J Rehabil Med. 1970;2:92-8.

23. Dickhuth $\mathrm{H}-\mathrm{H}$, Huonker M, Münzel T, Drexler H, Berg A, Keul J. Individual anaerobic threshold for evaluation of competitive athletes and patients with left ventricular dysfunction. Adv Ergometry. 1991;173-9.

24. Heyward VH, Gibson L. Advanced fitness assessement and exercise prescription. 7th ed. Champaign: Human Kinetics Publishing, Champaign Illinois; 2014. p. 2014.

25. WHO. Global recommendations on physical activity for health. World Health Organization. 2010
26. Rzewnicki R, Van den Auweele Y, De Bourdeaudhuij I. Addressing overreporting on the International Physical Activity Questionnaire (IPAQ) telephone survey with a population sample. Public Health Nutr. 2003;6(3):299-305.

27. Harun SN, Hennig S, Wainwright C, Klein K. A systematic review of studies examining the rate of lung function decline in patients with cystic fibrosis. Paediatr Respir Rev. 2016;20:55-66.

28. Hebestreit H, Hulzebos E, Schneiderman J, Karila C, Boas S, Kriemler S, Dwyer T, Sahlberg M, Urquhart D, Lands L, Ratjen F, Takken T, Varanistkaya L, Rücker V, Hebestreit A, Usemann J, Radtke T. Cardiopulmonary exercise testing provides additional prognostic information in cystic fibrosis. Am J Respir Crit Care Med. 2019;199(8):987-95.

29. Cox NS, Alison JA, Button BM, Wilson JW, Holland AE. Feasibility and acceptability of an internet-based program to promote physical activity in adults with cystic fibrosis. Respir Care. 2015;60(3):422-9.

30. Chen JJ, Cooper DM, Haddad F, Sladkey A, Nussbaum E, Radom-Aizik S. Tele-exercise as a promising tool to promote exercise in children with cystic fibrosis. Front Public Heal. 2018;6(269):1-5.

31. Del Corral T, Cebrià Iranzo MÀ, López-de-Uralde-Villanueva I, MartínezAlejos R, Blanco I, Vilaró J. Effectiveness of a home-based active video game programme in young cystic fibrosis patients. Respiration. 2018;95(2):87-97.

32. Hebestreit H, Lands LC, Alarie N, Schaeff J, Karila C, Orenstein DM, et al. Effects of a partially supervised conditioning programme in cystic fibrosis: an international multi-centre randomised controlled trial (ACTIVATECF): study protocol. BMC Pulm Med. 2018;18(1):1-11.

33. Lang RL, Wilson C, Stockton K, Russell T, Johnston LM. CyFiT telehealth: protocol for a randomised controlled trial of an online outpatient physiotherapy service for children with cystic fibrosis. BMC Pulm Med. 2019;19(1):1-8.

34. Tomlinson OW, Shelley J, Trott J, Bowhay B, Chauhan R, Sheldon CD. The feasibility of online video calling to engage patients with cystic fibrosis in exercise training. J Telemed Telecare. 2020;26(6):356-64.

35. Iolascon G, Gimigliano F, Di Pietro G, Moretti A, Paoletta M, Rivezzi M, Distante A, Piscitelli P. Personalized paths for physical activity: developing a person-centered quantitative function to determine a customized amount of exercise and enhancing individual commitment. BMC Sports Sci Med Rehabil. 2021;13(1):60.

\section{Publisher's Note}

Springer Nature remains neutral with regard to jurisdictional claims in published maps and institutional affiliations.
Ready to submit your research? Choose BMC and benefit from:

- fast, convenient online submission

- thorough peer review by experienced researchers in your field

- rapid publication on acceptance

- support for research data, including large and complex data types

- gold Open Access which fosters wider collaboration and increased citations

- maximum visibility for your research: over $100 \mathrm{M}$ website views per year

At BMC, research is always in progress.

Learn more biomedcentral.com/submissions 